\section{Kognitive Kommunikationsstörungen}

\author{
Interview mit Dipl. Psych. Dr. rer. nat. Thomas Guthke
}

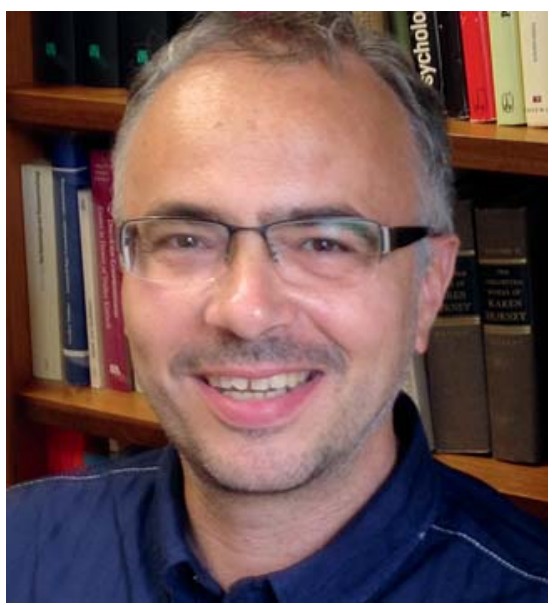

In der Rehabilitation neurologischer Patienten spielen nicht aphasische Kommunikationsstörungen eine große Rolle. Was versteht man darunter?

Der Begriff stammt ursprünglich von Prigatano (1985, Language Sciences), der mit einer nicht aphasischen Sprachstörung nach Schädel-Hirn-Trauma eine auffällige Kommunikation trotz weitgehender Rückbildung von linguistischen und kognitiven Defiziten beschrieb. Diese Patienten sind oft in herkömmlichen Tests unauffällig, sodass auch die Gefahr besteht, dass diese Schwierigkeiten in der Klinik nicht diagnostiziert bzw. zwischen Sprachtherapie und Neuropsychologie hin- und her geschoben werden.

Um Auffälligkeiten der Kommunikation, wie z. B. Abdriften oder Haften an Themen, thematische Inkohärenz, mangelnde Perspektivübernahme, unzureichende Berücksichtigung der Gesprächssituation oder des Gesprächspartners sowie Schwierigkeiten in der Textverarbeitung, zusammenzufassen, hilft das Konzept der Kognitiven Kommunikationsstörungen (z. B. Regenbrecht \& Guthke, 2017, Aphasie und verwandte Gebiete) weiter, welches sehr unterschiedliche Störungsprofile umfasst, aber herausstellt, dass die Kommunikationsschwierigkeiten durch ein Wechselspiel linguistischer, kognitiver und behavioraler Prozesse bestimmt sind.
Welchen Stellenwert haben dabei Exekutivfunktionen? Wie können darauf zurückzuführende Kommunikationsstörungen erkannt werden?

Eine zentrale Rolle für das Verständnis Kognitiver Kommunikationsstörungen spielen hierbei Störungen exekutiver Funktionen. Exekutive Funktionen werden in der Regel mit Steuerungs- oder Leitungsfunktionen übersetzt. Als exekutive Funktionen werden metakognitive Prozesse bezeichnet, die zum Erreichen eines definierten Zieles die flexible Koordination mehrerer Subprozesse steuern bzw. ohne Vorliegen eines definierten Zieles bei der Zielerarbeitung beteiligt sind. Insofern stellt eine erfolgreiche Kommunikation hohe Ansprüche an das Funktionieren dieser exekutiven Prozesse. Für Kommunikationsstörungen spielen außerdem noch Beeinträchtigungen der Fähigkeit der Theory of Mind eine relevante Rolle, also die Fähigkeit, bei anderen Personen z. B. deren Ideen, Absichten, Gefühle und Meinungen zu erkennen.

\section{Was muss bei der neuropsychologi-} schen und logopädischen Behandlung von Patienten mit kognitiver Kommunikationsstörung berücksichtigt werden?

Im klinischen Umgang erfordern die Störungsbilder der Kognitiven Kommunikationsstörungen eine interdisziplinäre Vorgehensweise der Neuropsychologie und Sprachtherapie, um die Kernsymptomatik zu beschreiben, relevante funktionelle Ursachen zu erfassen sowie angemessene Therapieziele und Therapiemethoden festzulegen. Ohne eine entsprechende neuropsychologische Diagnostik sind die Symptome der Kognitiven Kommunikationsstörung nicht sicher interpretierbar. Ebenso obligatorisch ist eine Diagnostik sprachsystematischer und sprechmotorischer Leistungen, weil Patienten mit Kognitiven Kommunikationsstörungen auch akute oder anhaltende Beeinträchtigungen vor allem auf lexikalisch-semantischer Ebene zeigen können.
Welche Erfahrungen liegen bei Patienten Kognitiven Kommunikationsstörungen im Hinblick auf Prognose und berufliche Wiedereingliederung vor? Entsprechende Auffälligkeiten in der Kommunikation können sich gravierend auf die berufliche Wiedereingliederung auswirken. Zum einen spielen kommunikative Anforderungen bei vielen Tätigkeiten eine zentrale Rolle, zum anderen sind sie bedeutsam für Absprachen mit Kollegen und Vorgesetzten. Positive Erfahrungen haben wir in der Therapie damit gemacht, in Vorbereitung und bei der Begleitung der beruflichen Reintegration berufsrelevante Kommunikationsübungen in Form von Rollenspielen zu solchen Themengebieten wie die Annahme von Hilfe, das Anerkennen von Leistungsgrenzen, das Informieren über berufsrelevante Erkrankungsfolgen und Funktionseinschränkungen sowie vorhandenen beruflichen Ressourcen mit einzubeziehen.

Das Interview führte Dr. Bruno Fimm, RWTH Aachen.

\section{ZUR PERSON}

Thomas Guthke studierte an der Humboldt-Universität zu Berlin Klinische Psychologie und promovierte auf dem Gebiet der kognitiven Psychologie. Als Postdoktorand verbrachte er 2 Jahre am Institut für Kognitionswissenschaft, University of Colorado at Boulder, USA. Von 1995 bis 2014 arbeitete er als Klinischer Neuropsychologe an der Tagesklinik für Kognitive Neurologie am Universitätsklinikum Leipzig. Aktuell ist er als Neuropsychologe und Verhaltenstherapeut in eigener Praxis mit Standorten in Wermsdorf und Leipzig niedergelassen. Seine Schwerpunkte liegen auf den Gebieten Kognitive Kommunikationsstörungen, berufliche Wiedereingliederung und neuropsychologische Psychotherapie. 